\title{
Hemotórax: etiología, diagnóstico, tratamiento y complicaciones
}

Arturo Cortes-Telles ${ }^{1}$, Carlos Enrique Morales-Villanueva ${ }^{2}$, Esperanza Figueroa-Hurtado $^{3}$

${ }^{1}$ Laboratorio de Fisiología Pulmonar. Departamento de Neumología. Hospital Regional de Alta Especialidad de la Península de Yucatán. ${ }^{2}$ Departamento de Neumología. Centro Médico La Raza (IMSS). ${ }^{3}$ Neumología Pediátrica. Unidad de Cuidados Intensivos Pediátricos. Hospital Regional de Alta Especialidad de la Península de Yucatán.

\section{Autor para correspondencia}

Nombre: Arturo Cortes-Telles

Institución: Laboratorio de Fisiología Pulmonar.

Departamento: Departamento de Neumología. HRAE Yucatán.

Dirección: Calle 7 No. 433 por 20 y 22. Fracc. Altabrisa. CP 97130.

E-mail: dr_morenheim@hotmail.com

Recibido: el 7 de agosto de 2015 Aceptado para publicación: el 25 de noviembre de 2015

Copyright @ 2016 por autores (s) y Revista Biomédica.

Este trabajo esta licenciado bajo las atribuciones de la Creative Commons (CC BY).

http://creativecommons.org/licenses/by/4.0/ 


\section{Resumen}

La sospecha de hemotórax inicia con una historia clínica adecuada, particularmente, el padecimiento actual, por ejemplo, casos con trauma torácico. El paso inicial de la evaluación, es diferenciar los derrames pleurales hemorrágicos de los verdaderos hemotórax. Confirmar el diagnóstico de manera temprana es fundamental, dado que, conforme progresan las fases de organización del coágulo, se van desarrollando adherencas entre la superficie del parénquima pulmonar y la pleura parietal, aspecto que dificulta evacuarlo mediante drenaje pleural convencional. La radiografía de tórax continúa siendo el estudio complementario inicial, sin embargo, es importante realizar estudios adicionales que permitan orientar la decisión terapéutica; la elección del análisis paraclínico puede justificarse con base en la experiencia y disponibilidad de recursos en el centro de atención. La instalación del tratamiento primario es crucial e inicia con el drenaje de la cavidad torácica vía sonda pleural en la mayoria de los casos; el uso de fibrinoliticos se considera de segunda línea y particularmente en hemotórax coagulado o casos que tienen riesgos significativos de complicaciones al someterse a un procedimiento quirúrgico mayor (Por ejemplo. decorticación). Las complicaciones se pueden disminuir al sistematizar el enfoque diagnóstico-terapéutico.

Palabras Clave: hemotórax, hemotórax coagulado, toracoscopía video-asistida, tratamiento, sonda pleural, trauma de tórax.

\section{Abstract}

\section{Hemothorax: etiology, diagnosis, treatment and complications}

Initial approach of cases with suspected hemothorax begins with a complete clinical history (eg. recent thoracic trauma). The first step is to differentiate hemorrhagic pleural effusion of true hemothorax; then, prompt diagnosis is essential. We must keep in mind, as time progresses, the clot firmly adheres to the lung and pleural surface making it difficult to treat; therefore, any delay in this process diminishes the opportunity to evacuate the hemothorax through conventional thoracostomy with chest tube insertion and pleural drainage. Chest X-ray still the initial study, however, complementary tests 
should be performed in order to guide therapeutic decisions. The choice must be justified on local availability and experience. Undoubtedly, primary evacuation is mandatory; fibrinolytics are reserved as second-line treatment, also can be considered in coagulated hemothorax or patients who are at a high risk of surgical complications.

In order to diminish any morbidity it is advisable to perform a systematic diagnostic and therapeutic approach.

Keywords: hemothorax, coagulated hemothorax, thoracic trauma, treatment, pleural drainage, video-assisted thoracoscopic surgery.

\section{DEFINICIÓN}

Hemotórax se define como la presencia de sangre en la cavidad pleural; no obstante, podemos encontrar líquido pleural de aspecto hemático durante la evaluación inicial de casos con derrame pleural. Cabe hacer mención, un nivel de hematocrito igual o mayor al $5 \%$ es suficiente para dar la apariencia hemática al líquido pleural; en estos escenarios, el diagnóstico de hemotórax se establece cuando el hematocrito en el líquido pleural corresponde a un valor igual o mayor al $50 \%$ del hematocrito de sangre periférica (1).

La identificación de los factores de riesgo que permitan distinguir los derrames hemorrágicos del hemotórax son cruciales para argumentar diagnósticos diferenciales.

\section{ETIOLOGÍA}

El trauma contuso es una causa frecuente de morbilidad y mortalidad a nivel mundial. En México, con base en cifras de la Organización Panaméricana de la Salud, fallecen anualmente 24 mil personas como consecuencia de accidentes de tránsito con una tasa de 14.4 decesos por 100,000 habitantes, ocupando el $7^{\circ}$ lugar a nivel mundial (2). Datos de centros especializados en atención de pacientes con trauma, informan que 1 de cada 4 muertes ocurren por lesiones de la caja torácica, de los cuales, $70 \%$ son ocasionados por accidentes de vehiculos con motor. Además, $60 \%$ de los casos reportados con politrauma presentan lesiones en el tórax (3).

Se ha informado previamente la existencia de un incremento gradual en la asociación entre el número de costillas fracturadas con el desarrollo de hemotórax (6\% sin fracturas costales, $24 \%$ con $1-2$ fracturas y $81 \%$ con más de 2 fracturas) (4).

Otras causas de hemotórax traumático incluyen: a) caídas de altura, b) lesiones por mecánica de aplastamiento del tórax y c) heridas por arma de fuego ó arma blanca que penetren el tórax (5). 
La etiología iatrogénica representa la segunda causa de hemotórax. Los factores de riesgo reconocidos incluyen: a) colocación de un acceso vascular (subclavia o yugular) y b) punciones pleurales diagnósticas o terapéuticas (1).

El hemotórax espontáneo ocurre con menor frecuencia y generalmente es secundario a tumores malignos, uso de anticoagulantes, anormalidades congénitas de la vasculatura pleuropulmonar, endometriosis pulmonar, tromboembolismo con infarto pulmonar, neumotórax con adherencias pleurales (crónicos) y anormalidades hematológicas como la hemofilia (6).

Las neoplasias más comunes asociadas al hemotórax incluyen: schwanomas de la enfermedad de von Recklinghausen y los tumores que se originan de tejidos blandos como sarcomas ó angiosarcomas (7). El cáncer de pulmón es una causa rara de hemotórax, incluso cuando hay compromiso pleural (6).

Los casos relacionados con anticoagulación son muy raros y la mayoría de las veces ocurren dentro de la primera semana de tratamiento.(8) Finalmente, entre las causas vasculares podemos hacer una subdivisión con base en el tipo de circulación; las vinculadas con la circulación mayor e incluyen disección o ruptura aórtica (principal causa espontánea vascular), laceración de vasos intercostales, ruptura de adherencias pleurales, lesión diafragmática o de estructuras vasculares del mediastino, heridas por arma de fuego o arma blanca que penetren el tórax y lesiones por efecto de aceleracióndesaceleración de gran impacto $(3,6,9,10)$ Por otro lado, entre las relacionadas con la circulación menor, la causa más frecuente es la lesión de la vasculatura del parénquima pulmonar (3) (Cuadro 1). 
Cuadro 1 Principales causas de hemotorax

\begin{tabular}{|c|c|c|c|}
\hline Traumática & Espontánea & Coagulopatías & Misceláneas \\
\hline $\begin{array}{c}\text { Traumatismo } \\
\text { torácico } \\
\text { cerrado }\end{array}$ & Vascular & $\begin{array}{c}\text { Inducida por } \\
\text { medicamentos }\end{array}$ & $\begin{array}{c}\text { Hema- } \\
\text { topoyesis } \\
\text { extramedular }\end{array}$ \\
\hline $\begin{array}{c}\text { Traumatismo } \\
\text { torácico } \\
\text { penetrante, } \\
\text { incluyendo el } \\
\text { iatrogénico }\end{array}$ & $\begin{array}{c}\text { Aneurisma/dis } \\
\text { ección aortica }\end{array}$ & $\begin{array}{c}\text { Enfermedad } \\
\text { congénita }\end{array}$ & Exostosis \\
\hline & $\begin{array}{c}\text { Malformación } \\
\text { arteriovenosa } \\
\text { (Rendu-Osler- } \\
\text { Weber) }\end{array}$ & $\begin{array}{c}\text { Neoplasia } \\
\text { (primaria o } \\
\text { metastásica) }\end{array}$ & Endometriosis \\
\hline & $\begin{array}{c}\text { Enfermedad } \\
\text { aneurismática } \\
\text { (Elhers- } \\
\text { Danlos) }\end{array}$ & & \\
\hline infarto & & \\
\hline & $\begin{array}{l}\text { Embolia } \\
\text { pulmar con }\end{array}$ & \\
\hline
\end{tabular}

\section{CUADRO CLÍNICO}

Los síntomas y signos varían dependiendo de la causa, el volumen y velocidad de acumulación. En el hemotórax traumático agudo predominan las manifestaciones hemodinámicas (taquicardia, choque hipovolémico) y la presencia de dolor torácico. Por otro lado, casos de hemotórax no traumáticos manifiestan con mayor frecuencia disnea. En ambos escenarios clínicos existen signos de ocupación pleural (1). La adición de contusión pulmonar genera signos de consolidación (11).

En el hemotórax espontaneo, la historia clínica y examen físico pueden orientar hacia la etiología. Por ejemplo, antecedente de hemoptisis (tiene relación con malformaciones arteriovenosas), uso previo de medicamentos (antiagregantes plaquetarios, anticoagulantes), tendencia al sangrado por coagulopatia (asociación con epistaxis, hematuria, derrames articulares), periodicidad de los derrames (asociación con los ciclos menstruales en endometriosis pleural) entre otros (6).

\section{DIAGNÓSTICO}

Con base en la frecuencia de la entidad clínica, la sospecha de hemotórax debe considerarse en todo paciente que ingresa al servicio de urgencias con antecedente reciente de herida penetrante en tórax 
y/o trauma torácico ó toracoabdominal (3). Cabe recordar, el diagnóstico definitivo se obtiene con la cuantificación del hematocrito en el líquido pleural (1). No obstante, podemos respaldarnos de métodos auxiliares para el diagnóstico.

Radiografía de tórax. Es el estudio inicial de todo paciente con trauma torácico y sospecha de hemotórax (10). Se recomienda obtener proyecciones postero-anteriores, dado que resulta más complejo la evaluación con el paciente en decúbito supino, asimismo, puede subestimarse el volumen del hemotórax (12). En la fase aguda, los hallazgos son compatibles con derrame pleural, es decir, existe borramiento del ángulo costodiafragmático, elevación del hemidiafragma comprometido, desplazamiento del mediastino al hemitórax contralateral a la lesión, entre otros. Por otro lado, en las formas crónicas existen cambios propiamente en la pleura y las opacidades que se generan del compromiso pueden orientarnos a la presencia de loculaciones. Cabe recordar que se necesita un volumen de $200 \mathrm{ml}$ en el espacio pleural para borrar el ángulo costofrénico en la radiografía posteroanterior del tórax en posición vertical (5).

Las radiografías laterales en bipedestación o en decúbito lateral son más sensibles para detectar derrames pequeños (50-100 ml) (5).

Adicionalmente la radiografía del tórax permite detectar lesiones que orientan hacia la etiología del hemotórax, entre ellas: lesiones óseas y ensanchamiento mediastinal (10).

Ultrasonido. Es el estudio de imagen empleado por centros con experiencia en trauma durante la evaluación inicial de todo paciente con sospecha de hemotórax; particularmente resulta útil y confiable en situaciones clínicas que imposibilitan el traslado del paciente a estudios como tomografía de tórax. Brooks y colaboradores, analizaron 61 pacientes con trauma de tórax con el objetivo de establecer la confiabilidad del ultrasonido torácico, documentando una sensibilidad del $92 \%$ y especificidad del 100\% (13). Sin embargo, otros autores han informado algunos límites del ultrasonido, particularmente, que la confiabilidad diagnóstica no es superior a la tomografía de tórax y la falta de una imagen global de la anatomía torácica (14).

Tomografía axial computarizada (TAC). La TAC es complementaria a la radiografía de tórax. La solicitud oportuna de la TAC en el abordaje del paciente con trauma moderado o grave conlleva un incremento en el diagnóstico de hemotórax oculto; sin embargo, el análisis de costo-beneficio y el impacto clínico deberá aclararse en ensayos clínicos subsecuentes (9).

Las indicaciones más apropiadas para su solicitud incluyen a) pacientes con persistencia de opacidades en la radiografía simple de tórax después de haber recibido manejo con drenaje pleural; b) establecer si el líquido acumulado es significativo y c) identificación y cuantificación de 
colecciones localizadas en regiones torácicas de difícil acceso para drenarse por sonda endopleural (v.gr. región anterior o superior del tórax) (10).

Cabe recordar, la sangre acumulada en la cavidad pleural habitualmente tiene una densidad entre 35 y 70 Unidades Hounsfield (UH) (15); no obstante, Oikonomou y cols. reportan la precisión de identificar el tipo de sangre acumulada con base en la densidad medida por TAC; en sangre no coagulada la densidad oscila de 30 a $45 \mathrm{UH}$, adicionalmente, el realce del contraste sobre una densidad homogénea confirma la presencia de sangrado activo que resplada una intervención urgente, por otro lado, la sangre coagulada se encuentra entre 50 y $90 \mathrm{UH}(16)$.

En casos donde se confirma la presencia de un hemotórax oculto o de presentación no habitual, la TAC de tórax permite evaluar su volumen; si el diámetro es mayor a $1.5 \mathrm{~cm}$ de diámetro, tiene 4 veces más probabilidad de requerir drenaje pleural (17).

Del mismo modo, cuando se sospechan lesiones en mediastino o parénquima pulmonar, la TAC de Tórax ofrece información útil que facilita la toma de decisiones (9). En casos con sospecha de fistula arteriovenosa o malformación arteriovenosa, el abordaje diagnóstico debe incluir una angiotomografía axial computarizada o arteriografía (6). Visualizar el medio de contraste extravasado en el sitio del hemotorax sugiere sangrado activo y es indicación de intervención quirúrgica urgente (3).

Análisis del líquido pleural. Es imperativo obtener una muestra de líquido pleural en toda sospecha de hemotórax. El procedimiento de elección es la toracocentésis. En situaciones donde el volumen del hemotórax sea pequeño es recomendable la punción guiada por ultrasonido (14). En caso de no contar con ultrasonido, puede realizar la punción pleural auxiliándose de una radiografía de torax en decúbito lateral, siempre y cuando la distancia entre la línea horizontal del derrame pleural y la pared torácica sea superior a $1 \mathrm{~cm}$ (3). Por otro lado, escenarios clínicos que presenten de manera simultánea trombocitopenia menor a 50,000 células/mcL y desarrollen algún hemotórax, se aconseja tratar la comorbilidad previo al procedimiento diagnóstico (1).

Citología de líquido pleural. Es útil en el abordaje diagnóstico del hemotorax secundario a etiología neoplásica; cabe hacer mención, se recomienda solicitar marcadores de inmunohistoquimica para aumentar el rendimiento diagnóstico (6). El análisis citológico en otras entidades clínicas infrecuentes, como la endometriosis pleural, demuestran la presencia de histiocitos cargados con hemosiderina y en raras ocasiones, pueden identificarse las células del epitelio endometrial (6).

\section{TRATAMIENTO}


La clave del tratamiento se relaciona estrechamente con la etiología del hemotórax.

Hemotórax agudo. El manejo inicial debe enfocarse en la identificación de situaciones que comprometan la vida, control del sangrado y reanimación para controlar el estado hemodinámico (3).

En pacientes hemodinámicamente estables y con hemotórax pequeño (volumen menor o igual a 300 ml) puede optarse por vigilancia clínico-radiológica. Por otro lado, pacientes con inestabilidad hemodinámica y/o cuyo hemotórax tenga volumen calculado mayor de $300 \mathrm{ml}$, deberán someterse a drenaje pleural. La sonda debe dirigirse hacia la parte posterior del tórax para permitir el drenaje incluso en decúbito supino (1). El tubo torácico recomendado oscila entre 28 y 36 Fr (18). Esta sugerencia deriva del estudio de Inaba y cols. quienes incluyeron 293 pacientes con hemotórax agudo tratados en un centro de trauma. No observaron diferencias en eficacia del drenaje, tasa de complicaciones o escala análoga del dolor, cuando se utiliza un calibre 28-32 Fr versus 36-40 Fr (19). $\mathrm{Al}$ igual que otros escenarios clínicos donde se emplea rutinariamente el drenaje pleural, en casos con hemotórax agudo secundario a trauma se ha reportado la eficacia del drenaje al utilizar un catéter venoso central comparado con la sonda endopleural, sin encontrar diferencias en la evolución del drenaje. (20) Cabe destacar, los autores consideramos que esta información debe emplearse con cautela, dado que, deriva de un ensayo que requiere reproducirse previo a poder sugerir el uso rutinario.

En casos secundarios a ruptura de aneurisma aórtico, debemos enfatizar 2 aspectos: 1) realizar una TAC contrastada y 2) no está indicado el drenaje, dado que puede favorecer la exanguinación (1).

La utilidad del drenaje pleural se circunscribe a los siguientes puntos: (a) evacuar la sangre del espacio pleural disminuyendo la posibilidad de empiema y/o fibrotórax; (b) detener la hemorragia del parénquima o laceraciones pleurales por la interposición de superficies pleurales al crear un taponamiento y (c) proporcionar una medición cuantitativa del sangrado (3).

Después de colocar la sonda endopleural, se sugiere realizar una radiografía simple con el objetivo de identificar la posición del tubo torácico (específicamente para ratificar la colocación dentro del espacio pleural) (21); asimismo, poder identificar anormalidades intratorácicas previamente no visualizadas por el hemotórax $(3,9)$.

Cabe hacer mención, que 80-90 \% de los casos resuelven el hemotorax con la colocación de drenaje pleural (10).

Hemotórax masivo. Es una indicación de intervención quirúrgica. La definición de hemotórax masivo incluye: a) cuantificación de un volumen de sangre igual o mayor a 1.5 litros inmediatamente 
posterior a la colocación del drenaje pleural ó b) sangrado cuantificado en el drenaje a ritmo de 200 $\mathrm{ml} /$ hora por 4 horas consecutivas. No obstante, el estado hemodinámico es la condicionante principal para definir el mejor momento de la cirugía. La ruta de resolución sugerida es vía toracotomía anterior; no obstante, centros con gran experiencia sugieren el empleo de video-toracoscopía asistida (VATS) (3).

Trombolíticos intrapleurales. Los trombolíticos intrapleurales han demostrado su utilidad en derrame paraneumónico complicado y/o empiema (22). En casos con hemotórax, pueden ser utilizados para mejorar el drenaje cuando se documenta la fase de coagulación, pero la evidencia es limitada; los estudios disponibles se han realizado del $1^{\circ}$ al $30^{\circ}$ días después de la colocación de drenaje pleural con resultados satisfactorios (23); sin embargo, otros autores los recomiendan en el periodo subagudo (durante los primeros 6 a 13 días de evolución); la evidencia es controversial, dado que, los resultados provienen de series de casos pequeñas que carecen de controles y han mostrado dificultades para medir la mejoría del hemotórax con la intervención propuesta $(23,24)$. Por ello, se les considera como tratamiento de segunda línea con enfoque en pacientes que tienen riesgos significativos de someterse a un procedimiento quirúrgico $(9,23)$.

Entre los tromboliticos sugeridos se encuentran estreptoquinasa (250,000 UI/dosis), uroquinasa (100,000 UI/dosis) y activador tisular del plasminogeno (t-PA, lteplase, $50 \mathrm{mgs}$ diluidos en $100 \mathrm{ml}$ de solución salina $0.9 \%$ /dosis); todos pueden indicarse cada $24 \mathrm{hrs}$ y permanecer en cavidad pleural durante un tiempo máximo estimado de 4 horas $(25,26)$. No se han observado diferencias entre estreptoquinasa o uroquinasa (25). El tiempo de tratamiento varia de 2-10 días, no obstante, la mayoría de los estudios se han llevado a cabo con 5 días y resultados satisfactorios (23). La tasa de éxito, definida como ausencia de síntomas y resolución de imagen radiográfica de hemotorax puede llegar a ser hasta del $90 \%$ en algunas series (27).

Antibióticos. El uso de antibióticos como profiláxis en hemotórax es un tema para discutir (28). La mejor evidencia disponible respalda el uso para la prevención de empiema en los casos con hemotórax secundario a trauma penetrante que requirieron la colocación de sonda pleural (29). La selección del antibiótico debe estar dirigida a los patógenos causales más frecuentes (en general, bacterias gram positivas) (30,31). El tiempo recomendado no se ha definido con exactitud, puede optar por $24 \mathrm{hrs}$ o hasta el retiro de la sonda (32).

Embolización. Es una estrategia terapéutica reservada para casos de hemotórax secundario a anomalías vasculares (6).

\section{COMPLICACIONES}


Todo hemotórax debe ser evacuado en el momento del diagnóstico. El retraso en el manejo favorece la organización del coagulo, como consecuencia, la pleura se adhiere a la superficie pulmonar dificultando la resolución. Se estima que este proceso ocurre a partir del $7^{\circ}$ día, tiempo en el que existe proliferación de fibroblastos estimulando la sobreproducción de matriz extracelular que engrosa la membrana del coágulo (10).

Hemotórax retenido o "coagulado". Se define como la presencia de sangre en la cavidad pleural que no pudo evacuarse a través del drenaje convencional, del mismo modo, persiste visible la zona opaca o densa en la radiografía o TAC del tórax respectivamente (33).

Esta complicación se presenta en 5\% de los pacientes que ingresan por hemotórax traumático o hemoneumotorax; asimismo, es considerado el factor de riesgo principal para el desarrollo de empiema (34).

Los centros con mayor experiencia en el manejo de hemotórax establecen que se requiere un volumen estimado mediante TAC de tórax de $500 \mathrm{ml}$ para decidir la evacuación quirúrgica del hemotórax retenido $(12,35)$. Por otro lado, sugieren un manejo conservador en los casos donde el volumen estimado es igual o menor de $300 \mathrm{ml}(36)$.

La estrategia quirúrgica de elección en la actualidad para el drenaje de hemotórax coagulado es la VATS $(24,35,36)$. La justificación para emplear VATS como estrategia principal incluye: 1) disminución de la estancia intrahospitalaria, 2) menor costo de atención hospitalaria y 3) menor frecuencia de toracotomía por persistencia del hemotórax (10). Inclusive, existen reportes donde se ha empleado VATS en adultos mayores con resultados satisfactorios, por lo tanto, la edad no es un factor que contraindique el procedimiento (37).

El procedimiento de toracotomía se reserva para casos no resueltos por VATS o coexistencia de atrapamiento pulmonar (fibrotórax) (33).

Empiema. En etapas tempranas únicamente podemos encontrar la presencia de un exudado sin un predominio claro de la celularidad (15 a 30\% de los casos); se recomienda que durante la obtención del líquido pleural mediante toracocentésis se lleven a cabo tinciones de gram y cultivos para descartar la posibilidad de infección (3). La tasa de empiema secundario a hemotórax es del 5\%; no obstante, se incrementa entre 28 y $33 \%$ en los casos con hemotorax retenidos secundarios a trauma (36,38). Se han identificado múltiples factores de riesgo, entre ellos: lesiones abdominales concomitantes, drenaje pleural prolongado (mayor de 6 días), contaminación del espacio pleural en el momento de la lesión (trauma penetrante), estancia en unidad de cuidados intensivos, contusión pulmonar, necesidad de laparotomía, fracturas costales, índice de gravedad de lesiones mayor a 25, 
casos que requieren más de un procedimiento terapéutico (VATS, uso de tromboliticos, colocación de un segunda sonda endopleural) (36,38). Finalmente, el desarrollo de fibrotórax ocurre en menos del $1 \%$ de los casos con hemotórax (3).

\section{CONCLUSIONES}

El hemotórax es una entidad clínica subestimada en nuestro medio, luego entonces, el diagnóstico temprano es clave. La evidencia es contundente y permite reconocer que la enfermedad conlleva un riesgo no despreciable de complicaciones que pueden poner en riesgo la vida de los pacientes o permancer con secuelas que incidan en una mala calidad de vida. Por lo tanto, debemos reforzar la sistematización diagnóstica en los casos que sean compatibles, el objetivo primario, realizar intervenciones que modifiquen el curso evolutivo adverso.

\section{Referencias}

1. Villena Garrido V, Ferrer Sancho J, Hernandez Blasco L, de Pablo Gafas A, Perez Rodriguez E, Rodriguez Panadero F, et al. [Diagnosis and treatment of pleural effusion]. Arch Bronconeumol. 2006 Jul; 42(7): 349-72.

2. Organización Panamericana de la Salud (OPS). http://www.paho.org/noticias/actualidad/mexico-ocupa el septimo-lugar-a nivel mundial muertes por-accidentes-de-transito-ops/20130101/nota/1818146.aspx. Fecha último acceso 21 Julio 2015.

3. Broderick SR. Hemothorax: Etiology, diagnosis, and management. Thorac Surg Clin. 2013 Feb; 23(1): 89-96.

4. Liman ST, Kuzucu A, Tastepe AI, Ulasan GN, Topcu S. Chest injury due to blunt trauma. Eur J Cardiothorac Surg. 2003 Mar; 23(3): 374-8.

5. Hooper C, Lee YC, Maskell N, Group BTSPG. Investigation of a unilateral pleural effusion in adults: British Thoracic Society Pleural Disease Guideline 2010. Thorax. 2010 Aug; 65 Suppl 2: 4-17.

6. Ali HA, Lippmann M, Mundathaje U, Khaleeq G. Spontaneous hemothorax: a comprehensive review. Chest. 2008 Nov; 134(5): 1056-65.

7. Tatebe S, Asami F, Shinohara H, Okamoto T, Kuraoka S. Ruptured aneurysm of the subclavian artery in a patient with von Recklinghausen's disease. Circ J. 2005 Apr; 69(4): 503-6.

8. Morecroft JA, Lea RE. Haemothorax--a complication of anticoagulation for suspected pulmonary embolism. Br J Clin Pract. 1988 May; 42(5): 217-8.

9. Freixinet-Gilart J, Hernandez-Rodriguez H, Martinez-Vallina P, Moreno-Balsalobre R, Rodriguez-Suarez P, Separ. Guidelines for the diagnosis and treatment of thoracic traumatism. Arch Bronconeumol. 2011 Jan; 47(1): 41-9.

10. Mowery NT, Gunter OL, Collier BR, Diaz JJ, Jr., Haut E, Hildreth A, et al. Practice management guidelines for management of hemothorax and occult pneumothorax. J Trauma. 2011 Feb; 70(2): 510-8.

11. Pasterkamp H, Kraman SS, Wodicka GR. Respiratory sounds. Advances beyond the stethoscope. Am J Respir Crit Care Med. 1997 Sep; 156(3 Pt 1): 974-87.

12. Velmahos GC, Demetriades D. Early thoracoscopy for the evacuation of undrained haemothorax. Eur J Surg. 1999 Oct; 165(10): 924-9.

13. Brooks A, Davies B, Smethhurst M, Connolly J. Emergency ultrasound in the acute assessment of haemothorax. Emerg Med J. 2004 Jan; 21(1): 44-6.

14. Rocco M, Carbone I, Morelli A, Bertoletti L, Rossi S, Vitale M, et al. Diagnostic accuracy of bedside ultrasonography in the ICU: feasibility of detecting pulmonary effusion and lung contusion in patients on respiratory support after severe blunt thoracic trauma. Acta Anaesthesiol Scand. 2008 Jul; 52(6): 776-84.

15. Kaewlai R, Avery LL, Asrani AV, Novelline RA. Multidetector CT of blunt thoracic trauma. Radiographics. 2008 
Oct; 28(6): 1555-70.

16. Oikonomou A, Prassopoulos P. CT imaging of blunt chest trauma. Insights Imaging. 2011 Jun; 2(3): 281-95.

17. Bilello JF, Davis JW, Lemaster DM. Occult traumatic hemothorax: when can sleeping dogs lie? Am J Surg. 2005 Dec; 190(6): 841-4.

18. Light RW. Pleural controversy: optimal chest tube size for drainage. Respirology. 2011 Feb; 16(2): 244-8.

19. Inaba K, Lustenberger T, Recinos G, Georgiou C, Velmahos GC, Brown C, et al. Does size matter? A prospective analysis of 28-32 versus 36-40 French chest tube size in trauma. J Trauma Acute Care Surg. 2012 Feb; 72(2): 4227.

20. Yi JH, Liu HB, Zhang M, Wu JS, Yang JX, Chen JM, et al. Management of traumatic hemothorax by closed thoracic drainage using a central venous catheter. J Zhejiang Univ Sci B. 2012 Jan; 13(1): 43-8.

21. Benns MV, Egger ME, Harbrecht BG, Franklin GA, Smith JW, Miller KR, et al. Does chest tube location matter? An analysis of chest tube position and the need for secondary interventions. J Trauma Acute Care Surg. 2015 Feb; 78(2): $386-90$

22. Cortes-Telles A, Laguna BP, Vázquez-Callejas A. Derrame paraneumónico y empiema. A propósito de una revisión integral. Evid Med Invest Salud. 2014 Jan-Mar; 7(1): 24-33.

23. Hunt I, Thakar C, Southon R, Bedard EL. Establishing a role for intra-pleural fibrinolysis in managing traumatic haemothoraces. Interact Cardiovasc Thorac Surg. 2009 Jan; 8(1): 129-33.

24. Oguzkaya F, Akcali Y, Bilgin M. Videothoracoscopy versus intrapleural streptokinase for management of post traumatic retained haemothorax: a retrospective study of 65 cases. Injury. 2005 Apr; 36(4): 526-9.

25. Skeete DA, Rutherford EJ, Schlidt SA, Abrams JE, Parker LA, Rich PB. Intrapleural tissue plasminogen activator for complicated pleural effusions. J Trauma. 2004 Dec; 57(6): 1178-83.

26. Thommi G, Nair CK, Aronow WS, Shehan C, Meyers P, McLeay M. Efficacy and safety of intrapleural instillation of alteplase in the management of complicated pleural effusion or empyema. Am J Ther. 2007 Jul-Aug; 14(4): 341-5.

27. Kimbrell BJ, Yamzon J, Petrone P, Asensio JA, Velmahos GC. Intrapleural thrombolysis for the management of undrained traumatic hemothorax: a prospective observational study. J Trauma. 2007 May; 62(5): 1175-78.

28. Moore FO, Duane TM, Hu CK, Fox AD, McQuay N, Jr., Lieber ML, et al. Presumptive antibiotic use in tube thoracostomy for traumatic hemopneumothorax: an Eastern Association for the Surgery of Trauma practice management guideline. J Trauma Acute Care Surg. 2012 Nov; 73(5 Suppl 4): 341-4.

29. Bosman A, de Jong MB, Debeij J, van den Broek PJ, Schipper IB. Systematic review and meta-analysis of antibiotic prophylaxis to prevent infections from chest drains in blunt and penetrating thoracic injuries. Br J Surg. 2012 Apr; 99(4): 506-13.

30. Sanabria A, Valdivieso E, Gomez G, Echeverry G. Prophylactic antibiotics in chest trauma: a meta-analysis of high-quality studies. World J Surg. 2006 Oct; 30(10): 1843-7.

31. DuBose J, Inaba K, Demetriades D, Scalea TM, O'Connor J, Menaker J, et al. Management of post-traumatic retained hemothorax: a prospective, observational, multicenter AAST study. J Trauma Acute Care Surg. 2012 Jan; 72(1): 11-22.

32. Boersma WG, Stigt JA, Smit HJ. Treatment of haemothorax. Respir Med. 2010 Nov; 104(11): 1583-7.

33. Morales Uribe CH, Villegas Lanau MI, Petro Sanchez RD. Best timing for thoracoscopic evacuation of retained post-traumatic hemothorax. Surg Endosc. 2008 Jan; 22(1): 91-5.

34. Karmy-Jones R, Holevar M, Sullivan RJ, Fleisig A, Jurkovich GJ. Residual hemothorax after chest tube placement correlates with increased risk of empyema following traumatic injury. Can Respir J. 2008 Jul-Aug; 15(5): 255-8.

35. Vassiliu P, Velmahos GC, Toutouzas KG. Timing, safety, and efficacy of thoracoscopic evacuation of undrained post-traumatic hemothorax. Am Surg. 2001 Dec; 67(12): 1165-9.

36. DuBose J, Inaba K, Okoye O, Demetriades D, Scalea T, O'Connor J, et al. Development of posttraumatic empyema in patients with retained hemothorax: results of a prospective, observational AAST study. J Trauma Acute Care Surg. 2012 Sep; 73(3): 752-7.

37. Schweigert M, Beron M, Dubecz A, Stadlhuber R, Stein H. Video-assisted thoracoscopic surgery for posttraumatic hemothorax in the very elderly. Thorac Cardiovasc Surg. 2012 Oct; 60(7): 474-9.

38. Eren S, Esme H, Sehitogullari A, Durkan A. The risk factors and management of posttraumatic empyema in trauma patients. Injury. 2008 Jan; 39(1): 44-9. 